\title{
Genetic Algorithm Based QoS Aware Adaptive Subcarrier Allocation in Cognitive Radio Networks
}

\author{
Dipak P. Patil, Vishal A. Wankhede, Vijay M. Wadhai \\ Sant Gadge Baba Amravati University, Amravat, India \\ Email: dipakpatil25@gmail.com, wankhedeva@gmail.com, vijay.wadhai@gmail.com
}

Received 9 April 2015; accepted 9 October 2015; published 12 October 2015

Copyright (C) 2015 by authors and Scientific Research Publishing Inc.

This work is licensed under the Creative Commons Attribution International License (CC BY). http://creativecommons.org/licenses/by/4.0/

(c) (i) Open Access

\section{Abstract}

In this paper, an adaptive subcarrier allocation scheme with reconfiguration of operating parameters for Cognitive Radio Networks (CRN) is presented. A QoS-conscious spectrum decision frame work is projected, where spectrum bands are determined by considering the application requirements as well as the dynamic nature of the spectrum bands. The novel subcarrier allocation algorithm is developed to fulfill different performance objective as a solution for subcarrier allocation and power allocation problem for Cognitive Radio (CR) users in CRNs. It employs operating frequency parameter modification using Proportional Resource Algorithm and Genetic Algorithm (GA). The multi objective optimization problem with equality and inequality constraint is considered. Moreover, a dynamic subcarrier allocations scheme is developed based on GA to decide on the spectrum bands adaptively dependent on the time-varying CR network capacity. The proposed algorithm targets to achieve maximum data rate for each subcarrier, maximize the overall network throughput and maximize the number of satisfied user under the constraints of bandwidth and guarantee Quality of Service (QoS) requirement from dynamic spectrum management (DSM) perspective. Moreover, it determines the best available channel.

\section{Keywords}

Cognitive Radio, Genetic Algorithm, QoS, Subcarrier Allocation

\section{Introduction}

The wireless frequency spectrum has been materialized as one of the most challenged civic goods in recent years. With severe demand and limited supply, spectrum management and the resolute allocation of spectrum are still 
the challenge with the existing spectrum allocation policies. These policies are derived from static model in which the allocation of the spectrum is managed by the various governmental agencies worldwide, like Federal Communication Commission (FCC). It leads to the underutilization of the accessible spectrum and waste of precious frequency resources [1]. The Dynamics of Spectrum Management presents a useful and contemporary approach for transitioning to new frameworks. A promising mechanism to improve the spectrum utilization by exploiting the spectrum opportunity is based on the CR concept. This new area of research foresees the development of CR networks to further improve spectrum efficiency by supporting new wireless users in existing crowded spectrum without causing harm to the existing user's performance [2]. Researchers have presented numerous spectrum management schemes; those are specifically designed for CRN. These schemes carry out spectrum management functions required for CRNs with performance objective to gratify challenges like interference avoidance, QoS awareness and seamless communication. The CR executes dynamic supervision of an external and internal radio environment, user behavior and network state. It uses the methodology from the environment and adjusts its internal states to statistical variations in the incoming RF stimuli by making analogous changes in operating parameters, avoiding interference with licensed or unlicensed users. These capabilities can be realized by dynamic spectrum management functionalities. Cognitive capability and reconfiguration are the two major characteristics of CR identified to address DSM functions [3].

Channel selection is an important part that enables the CR to choose the best channel among the pool of sensed channels. The CR must be capable of dynamically adjusting the physical layer parameters like operating frequency, bandwidth, power etc. After allocation of the channel to the CR user, in the meantime if the PU arrives, then the CR should suspend its operation, and reallocation of another channel for uninterrupted service to achieve better QoS is still the challenge [4] [5]. Such interrupted suspension in the transmission of the CR user can cause destroy the QoS. Even though the works in [6] [7], consider QoS requirements, the works still have insufficiency. In [8], the authors proposed a combined strategy to allocate channels and power with the QoSsupported objective to get maximum data rate to each user in cognitive networks, but this algorithm has high complexity. To deal with such disruptive scenarios, a new model is presented in [9] [10]. To address the factors discussed above, the focus must be on how to generate cognitive wireless networking problems as optimization problems from the perspective of resource allocation. The goal is to present a new perspective of wireless networking and resource allocation problems from the optimization point of view by developing robust channel selection scheme.

The significance of linear programming (LP) derives in part from its many applications and in part from the existence of good general-purpose techniques for finding optimal solutions [11] [12]. Practically LP has some restrictions; the objective and the constraints are rarely linear functions of the optimization parameters. Therefore paradigm has to shift for more generalized programming methods to handle complicated problems. A GA is a search technique which has adaption and automation to find approximate solutions for optimization with its multi objective optimization ability in CRN [13]. GA is employed to optimize multicarrier transceivers and single software defined radio. Numerous channel selection methods using the adjustment of operating parameters like power, bit error rate (BER), bandwidth (BW) as the basic genes. In these schemes, the objective function meets to the optimal value and then termination condition is achieved based on the desired criteria. According to the schemes proposed in [14], the attention is given to minimizing the BER, maximizing power and throughput by describing the comprehensive simulation model for the parameter reconfiguration using diverse GA parameters to satisfy QoS constraint. However still all the schemes described are not provided the solution of in accommodating the effects of the PU activity on the performance of the CRN.

The remainder of the paper is organized as follows. Section 2 presents the system model. The GA, its applications and its modeling regarding CRN are presented in Section 3. An experimental result explains the experimental setup and simulation results of our proposed scheme in Section 4. Finally conclusions are drawn in Section 5.

\section{Problem Formulation}

The system model is built by considering CR network as shown in Figure 1. Number of CR users consider from $n=1,2,3, \cdots, N$. All CR users sporadically senses radio environment and collect information PUs operating under PU base station. Generalized Likelihood Ratio Test (GLRT) detection approach is considered for spectrum sensing which utilize the eigenvalues that are associated with noise subspace of the sample covariance matrix of the received signal vector hence, is more robust. It shows better probability of detection for large number of sensors with unknown parameters, and does not require any knowledge of the noise power or the signal power. 


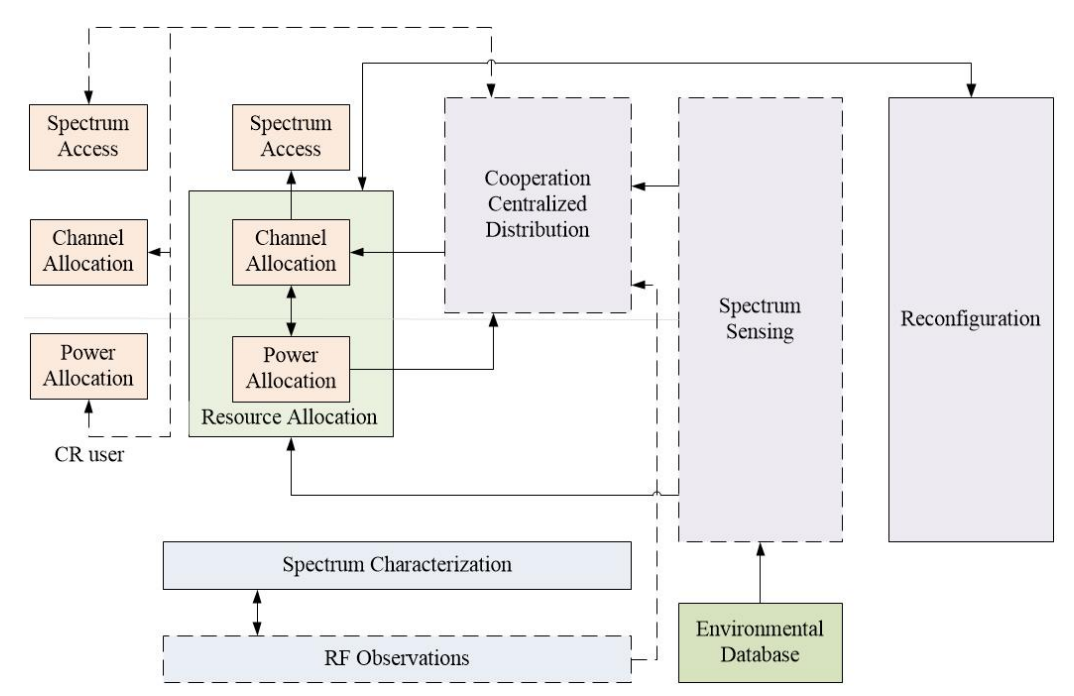

Figure 1. Proposed system model.

The proposed scheme initially detects the presence of PU transmitter reliability which is an important requirement as the considered network operates with multiple PU networks. Quick RF parameter adaption is prime objective of the proposed scheme.

With reference to [15] the GLRT sensing method considered using the intrinsic signal structure and treating the noise variance as an unknown parameter. ML parameter estimation underlying the GLRT scheme is also inspected. The more general form of the GLRT for the problem of interest can be written as,

$$
T_{G L R T}=\frac{\max _{h, \sigma_{s}^{2}} P\left(X \mid H_{1}, h, \sigma_{s}^{2}\right)}{\max _{\sigma_{s}^{2}} P\left(X \mid H_{0}, \sigma_{s}^{2}\right)}
$$

where, $P\left(X \mid H_{1}, h, \sigma_{s}^{2}\right)$ and $P\left(X \mid H_{0}, \sigma_{s}^{2}\right)$ represents likelihood functions under $H_{1}$ and $H_{0}$ respectively as,

$$
\begin{gathered}
P\left(X \mid H_{0}, \sigma_{s}^{2}\right)=\prod_{n=0}^{N-1} \frac{1}{\pi^{M \sigma_{s}^{2 M}}} \exp \left[-\frac{X^{H}(n) X(n)}{\sigma_{s}^{2}}\right] \\
P\left(X \mid H_{1}, h, \sigma_{s}^{2}\right)=\prod_{n=0}^{N-1} \frac{1}{\pi^{M\left|h^{H}+\sigma_{s}^{2} I\right|}} \times \exp \left[\left(-X^{H}(n)\left(h h^{H}+\sigma_{s}^{2} I\right)\right)^{-1} X(n)\right]
\end{gathered}
$$

Based on the sensing results obtained every CR user maintains a database of channel history as,

$$
\Phi_{s}^{k}=\left[\Phi_{s}^{1}, \Phi_{s}^{2}, \Phi_{s}^{3}, \cdots, \Phi_{s}^{k}\right]
$$

where, $\Phi_{s}^{k}$ is a PU activity vector during time slot $s$ and $k$ indicates particular channel.

$$
\Psi_{s}^{k}=\left[\Psi_{s}^{1}, \Psi_{s}^{2}, \Psi_{s}^{3}, \cdots, \Psi_{s}^{k}\right]
$$

where, $\Psi_{s}^{k}$ specifies channel history vector of a particular channel $k$ for $s$ number of time slot. The value of $s$ considered is 100 .

$$
H=\left\{\begin{array}{cccc}
\Psi_{1}^{1} & \Psi_{2}^{1} & \ldots & \Psi_{s}^{1} \\
\Psi_{1}^{2} & \Psi_{2}^{2} & \ldots & \Psi_{s}^{2} \\
\vdots & \vdots & \ddots & \vdots \\
\Psi_{1}^{k} & \Psi_{2}^{k} & \ldots & \Psi_{s}^{k}
\end{array}\right\}
$$

The history matrix is denoted by $H$ for the PUs on the $k$ channels. It maintains history of each channel and 
based on this history the Transmission Possibility Scale (TPS) for each available channel is calculated.TPS indicates upcoming accessibility of channel for CR users without interfering PU. TPS is represented as,

$$
\eta_{s}^{k}=e^{-}\left(\frac{\sum_{s=1}^{T_{s}} \Psi_{s}^{k}}{T_{s}}\right) \text { for } \Psi_{s}^{k}>
$$

where $\eta$ indicate TPS and $\psi$ represents the history pattern of the PU on the channel $T_{s}$ number of time slots and it is calculated using (5). The value of summation varies from 0 to 1 while the TPS $(\eta)$ varies from 0.3697 to 1 . The variables $T_{s}$ and $s$ can be used interchangeably and these are used together to improve clarity. The TPS purpose is to minimize the interference and switching overhead in order to get better QoS. This can be used to select the best channel in terms of the CR QoS requirement.

\section{Genetic Algorithm Based Subcarrier Allocation with Parameter Reconfiguration in CRN}

GA uses three different operators i.e. selection, crossover and mutation to direct the population towards convergence of the global optimum. Naturally these initial guesses are held as binary strings of the true variables, although an increasing number of GAs use real valued encoding. The subcarrier allocation with parameter reconfiguration (SAPR) system is centered around DSM with a hypothesis that inputs are provided either by sensing information from the radio environment or by the CR user. The CR senses the information from the environment which serves as initial population for the genetic algorithm (GA). After that the receiver engages in decision making process in order to provide new spectrum allocation demanded by the user. A new TPS gene inside the chromosome is added to facilitate the better QoS in terms of data rate, service time (delay) and minimal interruptions (channel switching). The TPS gene reduces number of retransmission because of collision with PU and accordingly saves the transmission power. The frequency band (FB), power (PWR), modulation method, (MOD) bit error rate (BER), data rate (DR) interference with primary user (IWPU) and TPS are the major genes considered, and the combination of these genes provide 30 bit solution (chromosomes).

After generation of population of chromosomes, the fitness evaluation of each chromosome in population is made, the fitness function $f_{i}$ is represented by,

$$
f_{i}=\left\{\begin{array}{lc}
{\left[\frac{w_{i} \cdot\left|x_{i}-x_{i}^{d}\right|}{x_{i}^{d}}\right],} & \text { if }\left|x_{i}-x_{i}^{d}\right|<x_{i}^{d} \\
w_{i}, & \text { otherwise. }
\end{array}\right.
$$

The fitness of chromosome is given by using " $w_{i}$ ” weight associated which varies as per the QoS requirement to the performance objective $f_{i}$.

$$
\begin{gathered}
F=\sum_{i=1}^{n} w_{i} \cdot f_{i} \\
=w_{d r} \cdot f_{d r}+w_{p w r} \cdot f_{p w r}+w_{b e r} \cdot f_{\text {ber }}+w_{\text {iwpu }} \cdot f_{\text {iwp }}+w_{T P S} \cdot f_{T P S}
\end{gathered}
$$

The percentage of total fitness is

$$
\text { Total fitness }(\%)=100\left[1-\sum_{i=1}^{n} w_{i} \cdot f_{i}\right]
$$

Probability of selection with each individual best chromosome from population, given by

$$
P_{i}=\frac{f_{i}}{\sum_{i=1}^{n} f_{i}}
$$

Subsequently the two point crossover process is carried out. Mutation process of forming the new generation is performed. Mutation is used to randomly flip the value of single bits within individual strings. After selection, crossover and mutation the string is applied to the initial population so that the new population can form the generational counter is incremented by one

The adaptive parameters of CR are symbolized as $x=\left(x_{1}, x_{2}, x_{3}, \cdots, x_{r}\right)$. FB, PWR, MOD, BER, IWPU, DR 
and TPS are the adjustable parameters. The objective functions are denoted as; $f=\left(f_{1}, f_{2}, f_{3}, \cdots, f_{q}\right)$, where $q$, corresponds to number of objective functions. The simplification process is performed by converting the multi objective optimization objective function in to single objective function by using the relationship specified. The formulation of objective function is given as,

$$
\begin{gathered}
f_{D R}=f(x)= \begin{cases}\frac{d_{r}-d_{c}}{d_{r}}, & d_{r}<d_{c} \\
1, & \text { otherwise }\end{cases} \\
f_{P W R}=1-\frac{P}{P_{\max }} \\
f_{B E R}=f(x)= \begin{cases}\frac{\log _{10}\left(\bar{P}_{b e}\right)}{\log _{10}\left(\bar{P}_{\text {minber }}\right)}, & P_{b e}<10^{-3} \\
1, & \text { otherwise }\end{cases} \\
f_{I W P U}=1-\frac{I}{I_{\max }} \\
f_{T P S}=\frac{\eta}{\eta_{\max }}
\end{gathered}
$$

where $d_{r}$, represents data rate and $d_{c}$ indicates the data rate available on the channel. $P$, indicates transmission power on a give channel and $P_{\max }$ maximum power available to the CR user. Average BER is represented by $\bar{P}_{\text {minber }}$ is the minimum value of BER. $I_{\max }$, indicates maximum interference limit to the PU. $\eta$ is the full opportunity for the CR on the given channel and $\eta_{\max }$ represents the maximum value of the opportunity index. The average power factor $(\mu)$ representing the power required to transmit the data in a given time slot.

The GA implementation process is summarized as below (Figure 2).

\section{Simulation Results}

In the proposed work of subcarrier allocation the CRN is considered, where each CR has capability of precise sensing and history management. The results are true for more generic cases. The GA parameter settings considered for implementation is as DR set to value of $265 \mathrm{kbps}$, PWR is $15 \mathrm{dBm}$ and BER is $10^{-5}$. For verification of operation the GA was modeled using RF parameters/genes. Several simulations were run and results are verified. The output consisted of final optimized gene values.

The simulation results are repeated 100 times with the 200 GAs iterations and the best chromosome is selected for checking the performance of objective function. To demonstrate the effectiveness the, proposed SAPR scheme is compared with random channel selection schemes.

The QoS requirement set for testing as FB $=400 \mathrm{MHz}, \mathrm{MOD}=\mathrm{QPSK}, \mathrm{BER}=10^{-9}$, PWR $=3 \mathrm{dBm}$, IWPU $=$ 0.25 , TPS $=0.9394$. The results obtained are shown in figures below. GA setting considered as, population size $=12$, crossover $=0.5$, mutation rate $=3 \%$, length of chromosomes $=32$, Elitism Rate $=2 \%$ and Number of generations $=100$. The 100 iterations are performed to find out the best solution TPS accomplish its optimal value and hence provides the required level of DR and other objectives mentioned above.

Table 1 summarizes the gene structure considered for GA implementation.

Four different modes are constituted to compute and plot the average objective function values to test the effectiveness of proposed scheme. Different objectives are addressed for different application and situation in each mode. Maximization of average throughput for CR user is the objective in High Throughput (HT) mode. This mode is suitable higher data rates applications. The Power responsive (PR) mode is effective for power sensitive application and suitable for the CR sensor networks and text messaging. The BER mode helps to reduce the BER for different applications like emergency applications. The QoS mode helps to reduce number of interruptions/channel switching with PUs. This mode also have power saving by avoiding the retransmission because of the collision occurs with the PU. Assumption is made that fitness function is equally dependent on all 


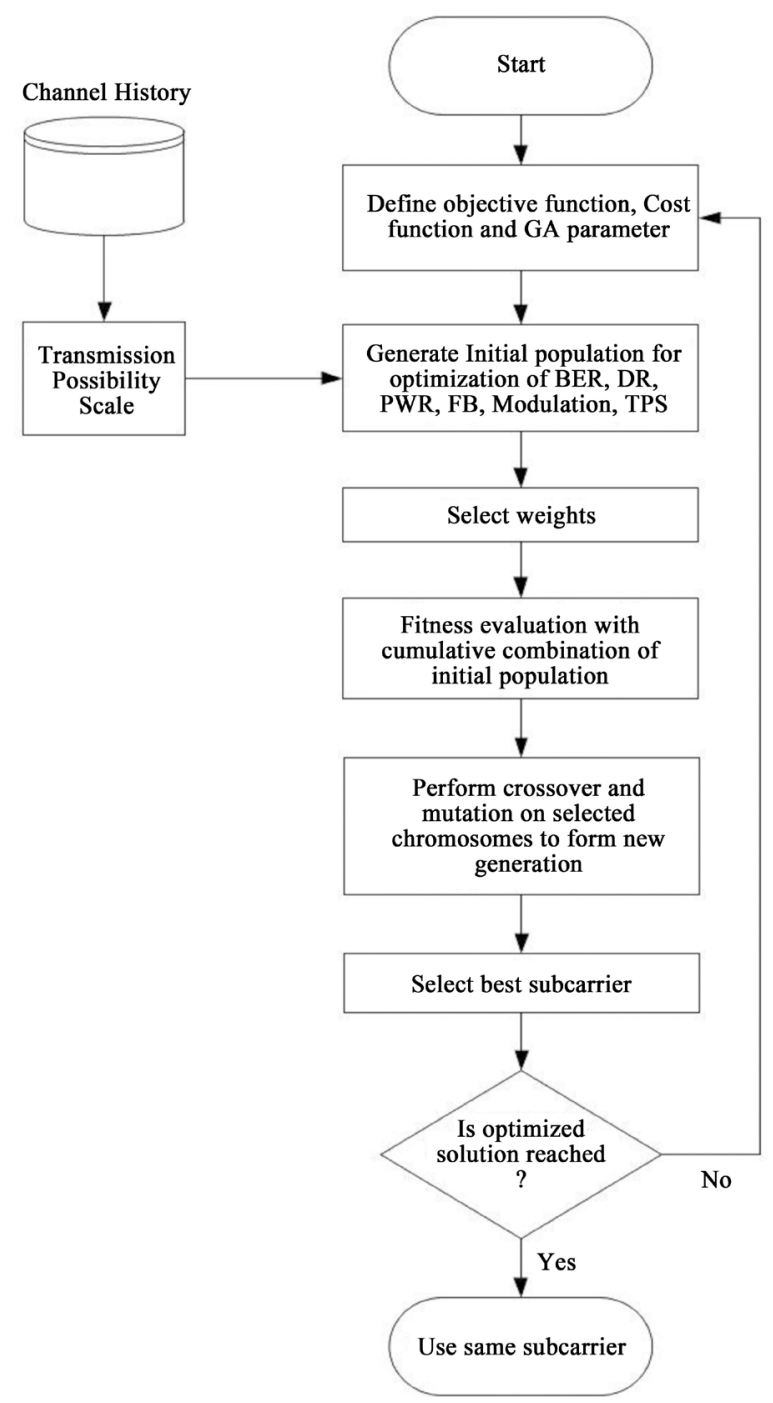

Figure 2. GA process flow chart for subcarrier allocation.

Table 1. Gene structure.

\begin{tabular}{cccc}
\hline Gene & Bits required & Range & Levels \\
FB & 7 & $54-862 \mathrm{MHz}$ & 100 \\
MOD & 2 & BPSK,QPSK, & 4 \\
BER & 4 & 8-QAM,16-QAM & 16 \\
DR & 5 & $10^{-1}-10^{-16}$ & 32 \\
PWR & 4 & $50-1.65 \mathrm{Mbps}$ & 16 \\
IWPU & 4 & $3-48 \mathrm{dBm}$ & 16 \\
TPS & 4 & $0.0625-1$ & 16 \\
\hline
\end{tabular}

parameters and weights have been choosing accordingly, and they depend on the QoS required from the end application.

For the system model described, simulation experiment conducted considering total 64 number of subcarriers with time varying frequency selective Rayleigh channel.

The progress of the algorithm with and without mutation, as a function of generation is shown in Figure 3. 
Mutation has been included by visiting every bit in each new child string which throwing a random number between 0 and 1 if this number is less than 1/64 flipping the value of the bit. Best fitted individual is shown in Figure 4. Above example is simulated for 100 iterations. And results are exhibited for first 60 iterations. Best fitness and Mean fitness shown in Figure 3 against generations carried. The Best fitness is in the range of 1.4881 and the Mean fitness is in the range of 1.4882. Figure 4 shows number of variables Vs current best individual. The first variable specify expected data rate and second variable is channel date rate. Fitness of the optimal individual is 1 hence after 1 st iteration values of individuals shown in the range of 0 to 0.8 . Children populated by each individual are shown in Figure 5. Average distance between individuals for number of generations is shown in Figure 6. It is used to estimate the distance between parent and child and minimum distance achieved suggest that the optimum value is reached which is 0 , specifies the solution of the objective function.

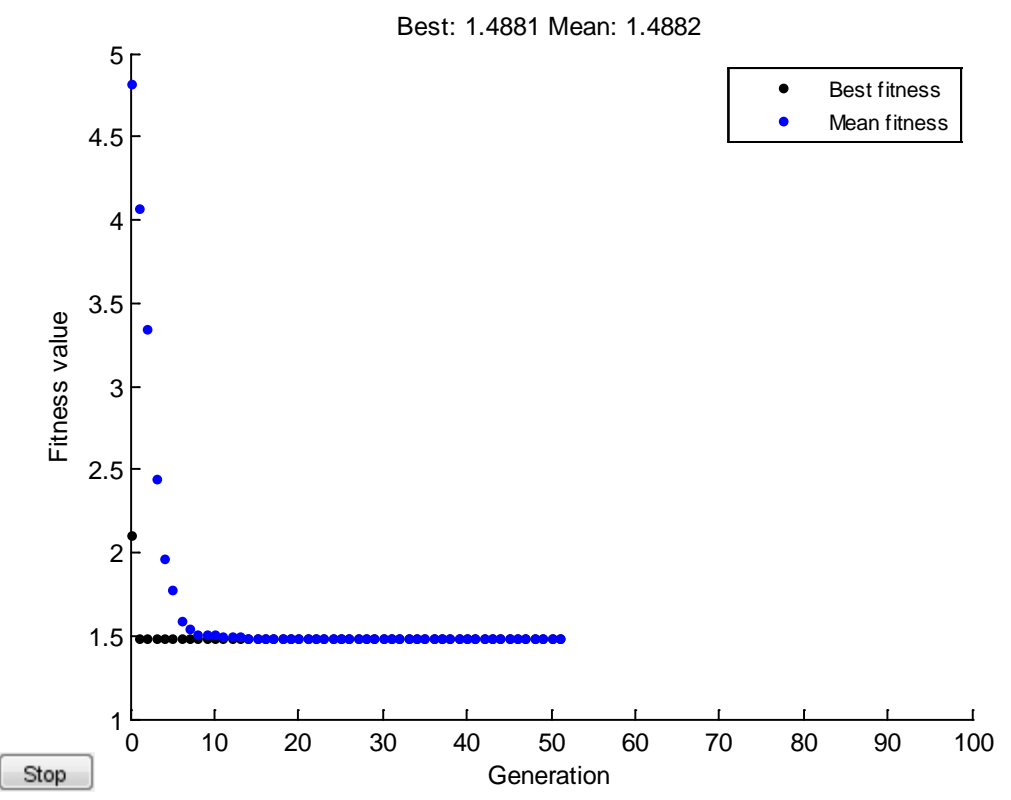

Figure 3. Fitness value vs. No. of generations.

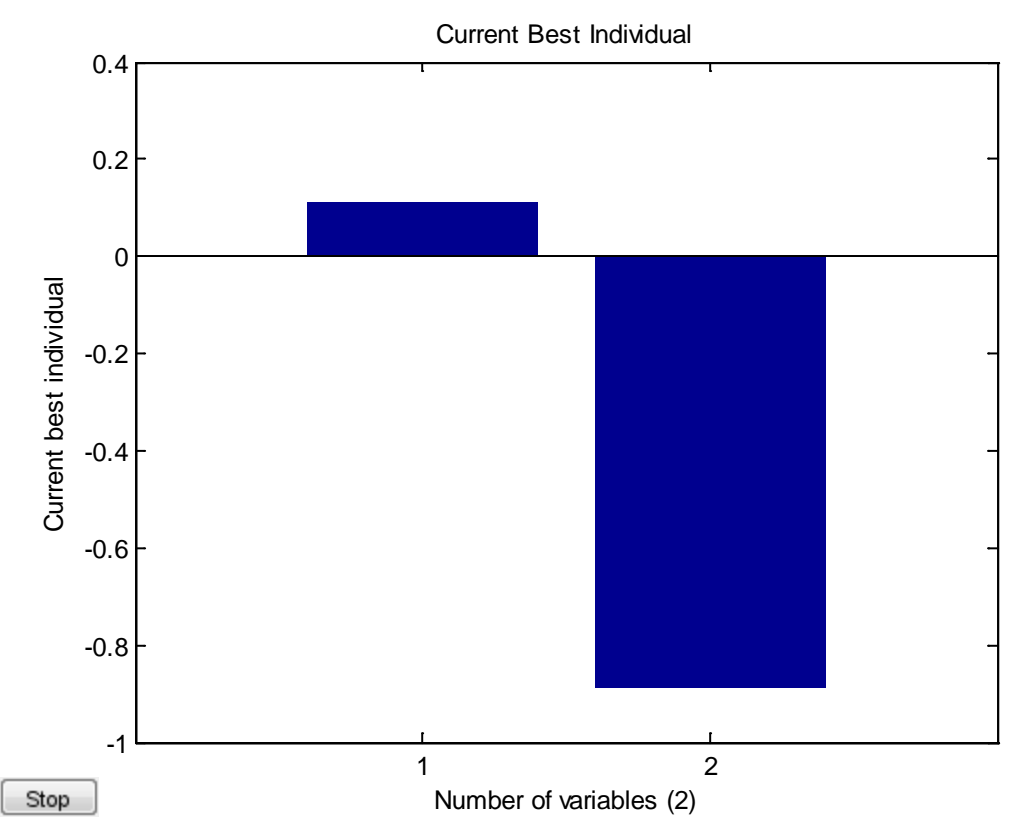

Figure 4. Best fitted individul vs No. of variables. 


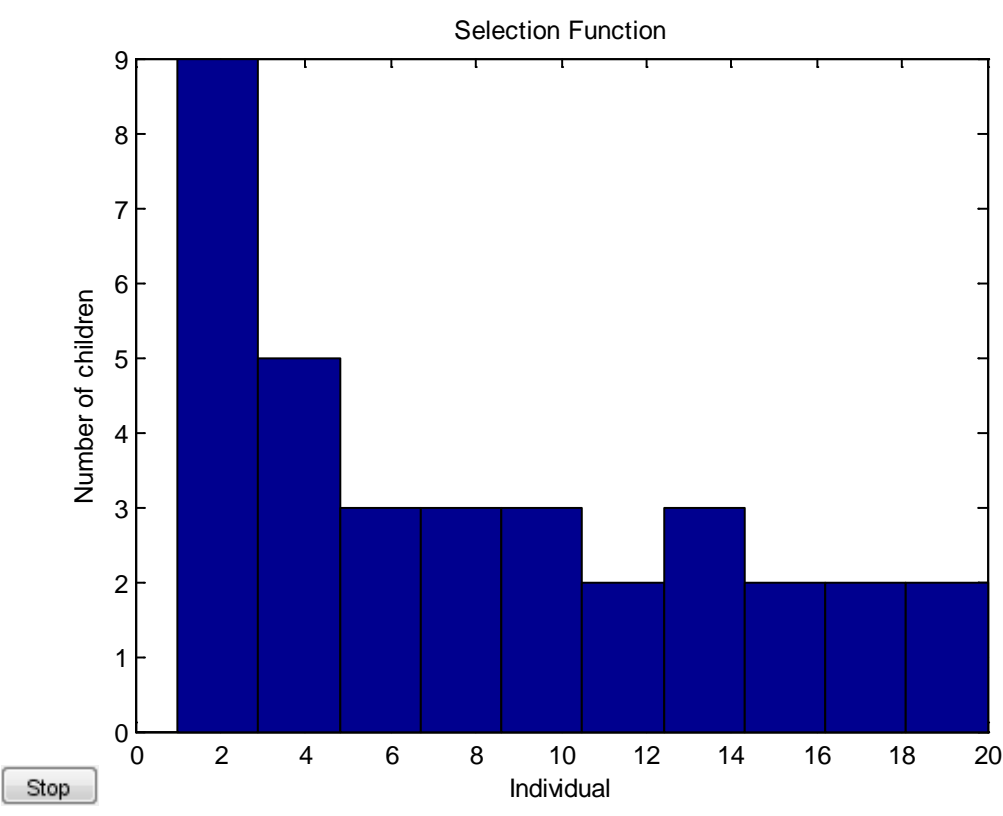

Figure 5. No. of children vs No. of individual.

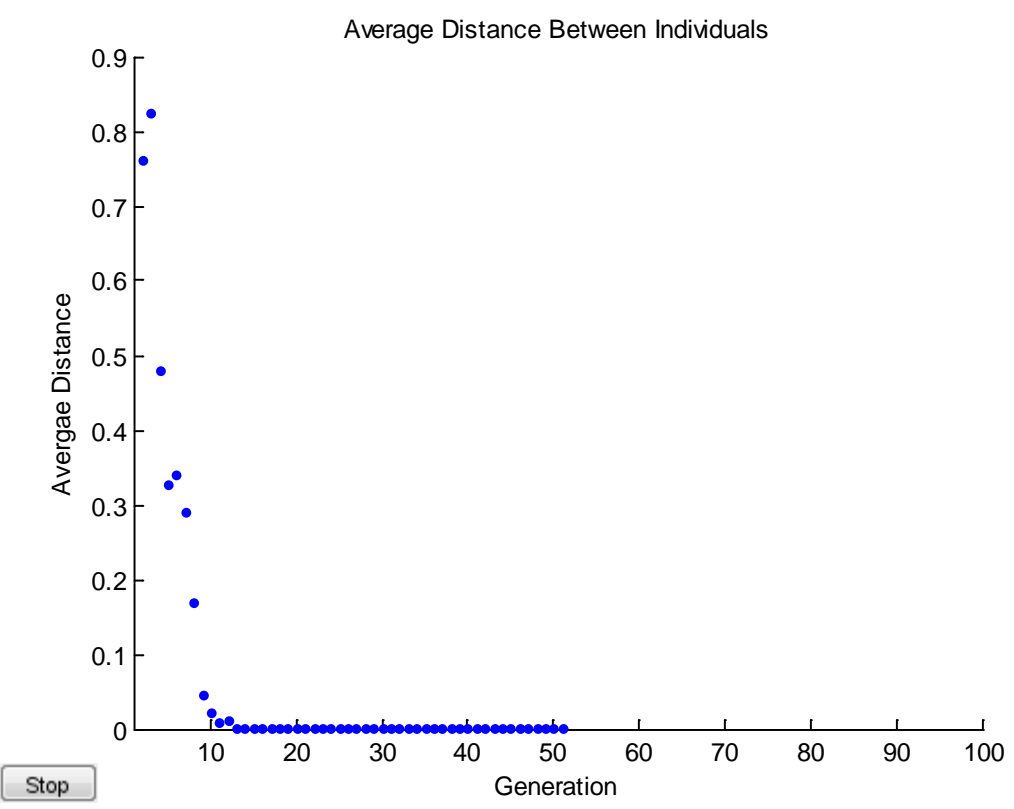

Figure 6. Average distance vs No. of generations.

Sample results for different modes of operation and value of relevant gene is presented in Figures 7-10. In HT mode which has the $65 \%$ weight. In this mode the data rate objective function reaches close to the optimal value of after 51 iterations. In PR mode, the objective function of minimizing power has maximum weight. In this mode the required power reduces in a few iterations. In this case $65 \%$ weight is given to the TPS gene which achieves its maximum value after 25 iterations and the rest of parameters are also approaching to their respective optimal values.

Figure 11 shows users with only best effort service, the capacity of each SU is evaluated over the different rate proportional. Results verify that GA guarantees to obtain the expectation capacity for a particular SU by adjusting the operating parameters. Figure 12 shows the performance of the proposed subcarrier allocation scheme over the number of users. As the number of users, the proposed scheme approach to the optimal solution 


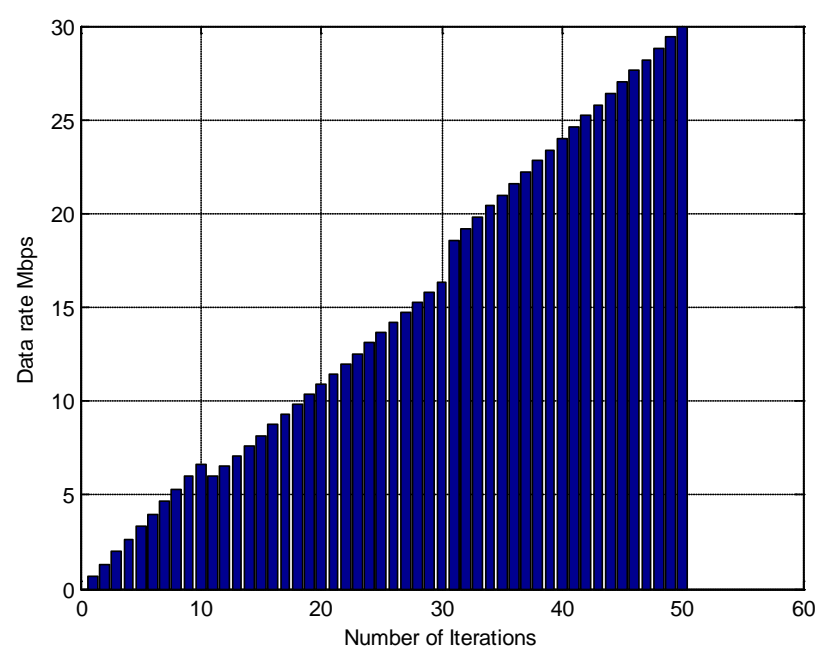

Figure 7. Data rate in DR mode.

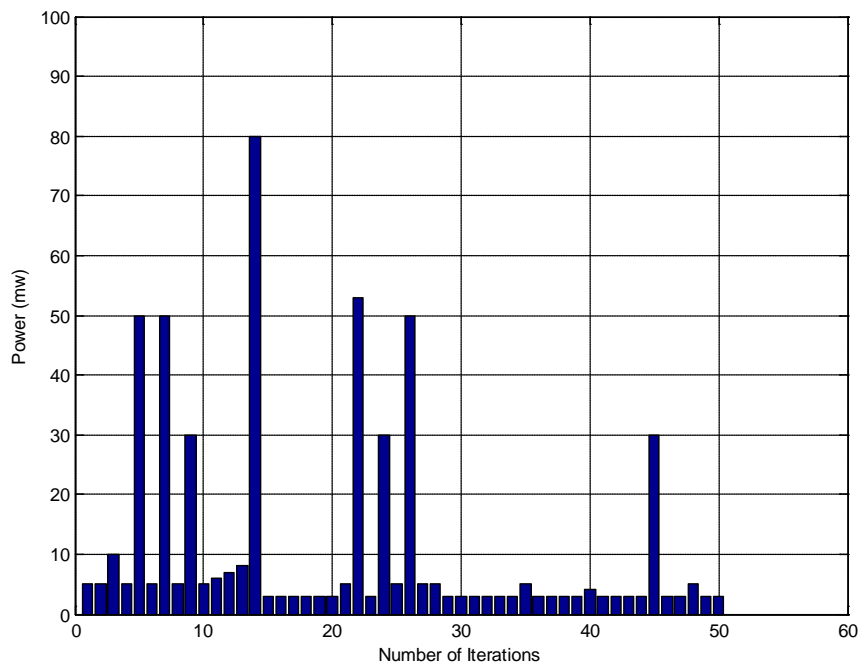

Figure 8. Power in PR mode.

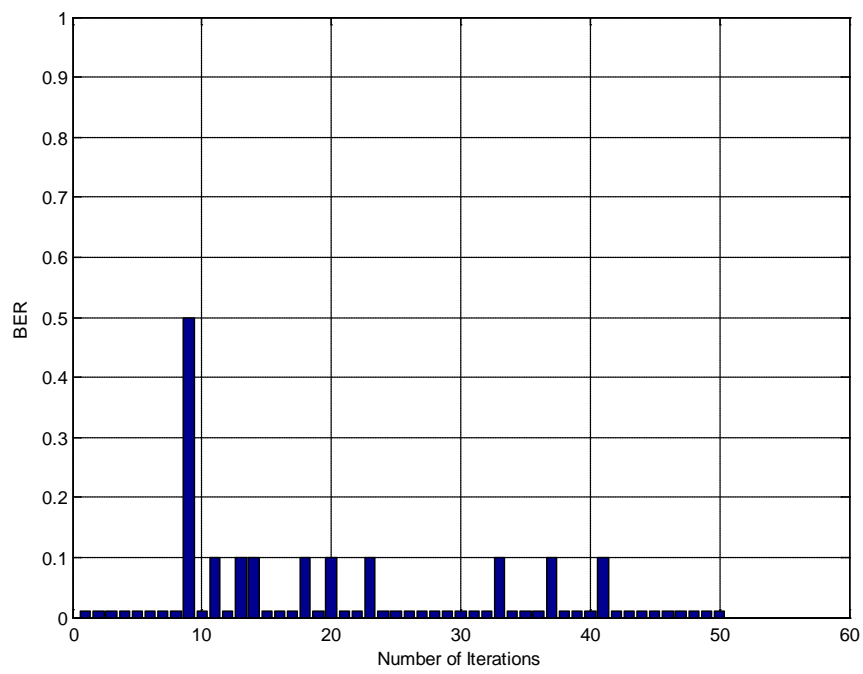

Figure 9. BER in BER mode. 


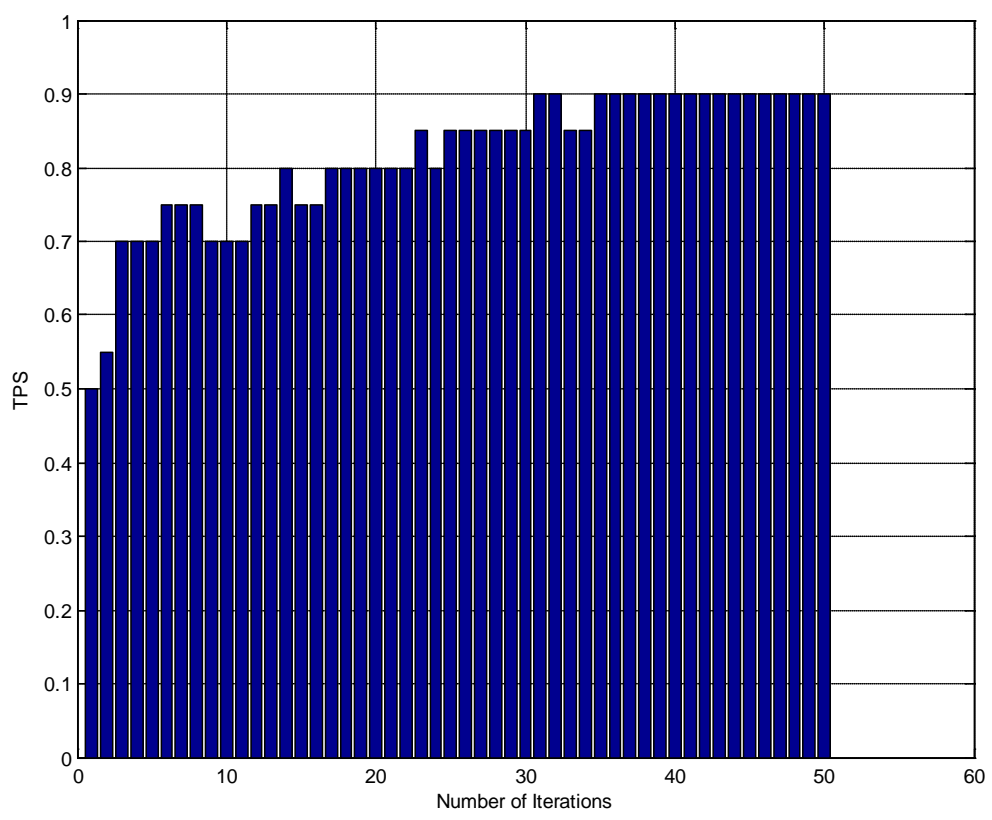

Figure 10. TPS in QoS mode.

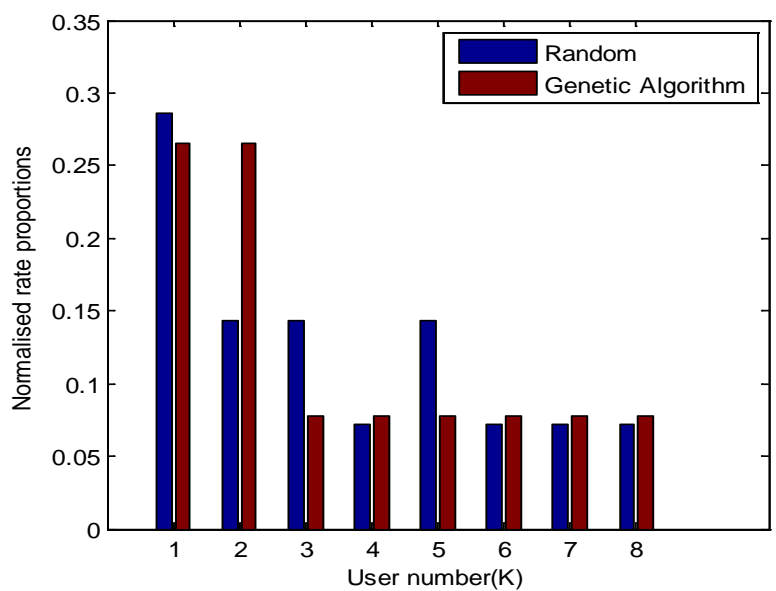

Figure 11. No. of users vs rate proportional.

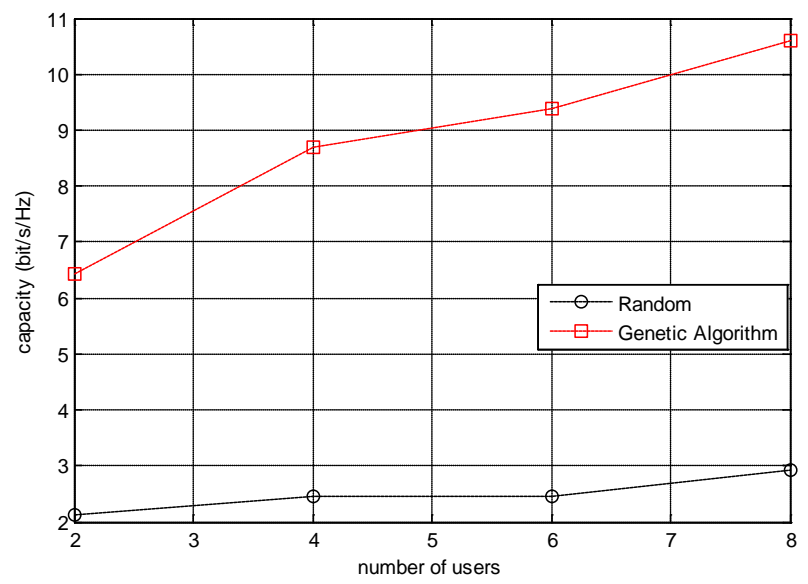

Figure 12. Channel capacity vs No. of users. 
with perfect channel information. The simulation results confirms that the information is fed back and association amongst subcarrier play a vital role in order to improve system performance. As compared to random scheme GA appears attractive as the number of users increases. Further it significantly reduces computational complexity and achieves higher capacities.

\section{Conclusion}

The proposed scheme addresses the major functions for CR user from spectrum decision perspective in CRN. The proposed scheme is based on GA and implemented considering all important issues counting channel characteristics, with assuring QoS issue without hampering PU operations. The future accessibility of channel for $\mathrm{CR}$ users without interfering PU is formulated, for selecting the optimal channel. The solution is developed through GA which performs genetic operations and then CR users reconfigure their parameters according to the allocated subcarrier. In connection with the weights given to particular objective functions different transmission modes are identified and their effectiveness is discussed. Results validate that, the fitness function of the individual parameters increases with increase in number of generations and reaches the optimum solution among the pool of available solution. All the parameters considered for fitness function are independent of each other. The major outcomes of the scheme are observed in the reduction in the number of channel switching, and the increase in channel capacity and data rate. The advantages make the SAPR scheme suitable for power sensitive applications like cognitive radio sensor network.

\section{References}

[1] Federal Communications Commission (2002) Spectrum Policy Task Force Report. ET Docket No. 02-135, November.

[2] Mitola, J. and Maguire, G.Q. (1999) Cognitive Radio: Making Software Radios More Personal. IEEE Personal Communications Magazine, 6, 6-13. http://dx.doi.org/10.1109/98.788210

[3] Akyildiz, I.F. (2011) Cooperative Spectrum Sensing in Cognitive Radio Networks: A Survey. Physical Communication, 4, 40-62. http://dx.doi.org/10.1016/j.phycom.2010.12.003

[4] Nie, C.C. (2005) Adaptive Channel Allocation Spectrum Etiquette for Cognitive Radio Networks. IEEE DySPAN, Baltimore, 8-11 November 2005, 269-278. http://dx.doi.org/10.1109/dyspan.2005.1542643

[5] Zheng, H. and Cao, L. (2005) Device-Centric Spectrum Management. IEEE DySPAN, 56-65.

[6] Hoang, A.T. and Liang, Y.-C. (2006) Maximizing Spectrum Utilization of Cognitive Radio Networks Using Channel Allocation and Power Control. IEEE 64th Vehicular Technology Conference, Montreal, 25-28 September 2006, 1-5. http://dx.doi.org/10.1109/vtcf.2006.257

[7] Zhang, L., Liang, Y.-C. and Xin, Y. (2007) Joint Admission Control and Power Allocation for Cognitive Radio Networks. IEEE International Conference on Acoustics, Speech and Signal Processing, Honolulu, 15-20 April 2007, III-673-III-676.

[8] Farah, J. and Marx, F. (2007) Combining Strategies for the Optimization of Resource Allocation in a Wireless Multiuser OFDM System. International Journal on Electronics Communication, 61, 665-677. http://dx.doi.org/10.1016/j.aeue.2007.01.002

[9] Yang, L., Cao, L. and Zheng, H. (2008) Proactive Channel Access in Dynamic Spectrum Networks. Physical Communication, 1, 103-111. http://dx.doi.org/10.1016/j.phycom.2008.05.001

[10] Marko, H., Sofie, P. and Aarne, M. (2008) Performance Improvement with Predictive Channel Selection for Cognitive Radios. IEEE CogART Workshop, 1-5.

[11] Wood, M.K. and Dantzig, G.B. (1949) Programming of Interdependent Activities: I, General Discussion. Econometrica, 17, 193-199. http://dx.doi.org/10.2307/1905522

[12] Dantzig, G.B. (1949) Programming of Interdependent Activities: II, Mathematical Model. Econometrica, 17, $200-211$. http://dx.doi.org/10.2307/1905523

[13] Qin, H., Su, J. and Du, Y. (2009) Multiobjective Evolutionary Optimization Algorithm for Cognitive Radio Networks. IEEE IEEC, Ternopil, 16-17 May 2009, 164-168. http://dx.doi.org/10.1109/ieec.2009.39

[14] Gardner, W. and Brown, W. (1987) Spectral Correlation of Modulated Signal. Part I-Analog Modulation. IEEE Transactions on Communications, COM-35, 584-594. http://dx.doi.org/10.1109/TCOM.1987.1096820

[15] Wang, P., Fang, J., Hang, N. and Lee, H. (2010) Multiantenna-Assisted Spectrum Sensing for Cognitive Radio. IEEE Transactions on Vehicular Technology, 59, 1791-1800. http://dx.doi.org/10.1109/TVT.2009.2037912 